\title{
An Integrated Approach to the Prediction of Chemotherapeutic Response in Patients with Breast Cancer
}

\author{
Kelly H. Salter ${ }^{1}$, Chaitanya R. Acharya ${ }^{1}$, Kelli S. Walters ${ }^{1}$, Richard Redman ${ }^{1,2}$, Ariel Anguiano ${ }^{1,2}$, \\ Katherine S. Garman ${ }^{1,2}$, Carey K. Anders ${ }^{1,2}$, Sayan Mukherjee ${ }^{1,3}$, Holly K. Dressman 1, William T. Barry ${ }^{1,3}$, \\ Kelly P. Marcom ${ }^{2}$, John Olson ${ }^{1,4}$, Joseph R. Nevins ${ }^{1}$, Anil Potti ${ }^{1,2 *}$ \\ 1 Duke Institute for Genome Sciences and Policy, Duke University, Durham, North Carolina, United States of America, 2 Department of Medicine, Duke University Medical \\ Center, Durham, North Carolina, United States of America, 3 Institute for Statistics and Decision Sciences, Duke University, Durham, North Carolina, United States of \\ America, 4 Department of Surgery, Duke University Medical Center, Durham, North Carolina, United States of America
}

\begin{abstract}
Background: A major challenge in oncology is the selection of the most effective chemotherapeutic agents for individual patients, while the administration of ineffective chemotherapy increases mortality and decreases quality of life in cancer patients. This emphasizes the need to evaluate every patient's probability of responding to each chemotherapeutic agent and limiting the agents used to those most likely to be effective.

Methods and Results: Using gene expression data on the NCl-60 and corresponding drug sensitivity, mRNA and microRNA profiles were developed representing sensitivity to individual chemotherapeutic agents. The mRNA signatures were tested in an independent cohort of 133 breast cancer patients treated with the TFAC (paclitaxel, 5-fluorouracil, adriamycin, and cyclophosphamide) chemotherapy regimen. To further dissect the biology of resistance, we applied signatures of oncogenic pathway activation and performed hierarchical clustering. We then used mRNA signatures of chemotherapy sensitivity to identify alternative therapeutics for patients resistant to TFAC. Profiles from mRNA and microRNA expression data represent distinct biologic mechanisms of resistance to common cytotoxic agents. The individual mRNA signatures were validated in an independent dataset of breast tumors $(P=0.002, N P V=82 \%)$. When the accuracy of the signatures was analyzed based on molecular variables, the predictive ability was found to be greater in basal-like than non basal-like patients $(P=0.03$ and $P=0.06)$. Samples from patients with co-activated Myc and E2F represented the cohort with the lowest percentage (8\%) of responders. Using mRNA signatures of sensitivity to other cytotoxic agents, we predict that TFAC non-responders are more likely to be sensitive to docetaxel $(P=0.04)$, representing a viable alternative therapy.
\end{abstract}

Conclusions: Our results suggest that the optimal strategy for chemotherapy sensitivity prediction integrates molecular variables such as ER and HER2 status with corresponding microRNA and mRNA expression profiles. Importantly, we also present evidence to support the concept that analysis of molecular variables can present a rational strategy to identifying alternative therapeutic opportunities.

Citation: Salter KH, Acharya CR, Walters KS, Redman R, Anguiano A, et al. (2008) An Integrated Approach to the Prediction of Chemotherapeutic Response in Patients with Breast Cancer. PLoS ONE 3(4): e1908. doi:10.1371/journal.pone.0001908

Editor: Toru Ouchi, Northwestern University, United States of America

Received December 21, 2007; Accepted February 22, 2008; Published April 2, 2008

Copyright: (C) 2008 Salter et al. This is an open-access article distributed under the terms of the Creative Commons Attribution License, which permits unrestricted use, distribution, and reproduction in any medium, provided the original author and source are credited.

Funding: The current work is supported by research grants from the Department of Defense (CDMRP) and the Jimmy V. Foundation.

Competing Interests: The authors have declared that no competing interests exist.

*E-mail: anil.potti@duke.edu

\section{Introduction}

One of the major challenges facing the field of oncology is the selection of the most effective chemotherapy agents for individual patients. While steps have been taken towards using biomarkers to select patients eligible to receive certain targeted therapies, selection of the more common cytotoxic agents remains largely arbitrary [1]. For example, patients with breast cancer may be given the "TFAC" (paclitaxel (T), 5-fluorouracil (F), adriamycin (A), and cyclophosphamide $(\mathrm{C})$ ) treatment regimen, DC (docetaxel and cyclophosphamide) treatment, or AC (adriamycin and cyclophosphamide) treatment in the neo-adjuvant setting, with little guidance as to which will actually be most effective for their particular disease. Furthermore, the administration of ineffective chemotherapy agents increases the probability of side effects and decreases the quality of life of many cancer patients[2,3], which further emphasizes the need to develop strategies that evaluate each individual patient's probability of responding to commonly used chemotherapeutic agents and limiting the agents used to those most likely to be effective. Recent advances in our understanding of cancer biology have offered a potential approach to meeting this challenge by using gene expression signatures of sensitive or resistant cell lines to predict patient response to a panel of commonly used chemotherapy agents $[4,5,6,7]$. These initial gene signatures, however, were created using U95 Av2 Affymetrix gene array chips, while in recent months the volume of usable data 
has shifted to include the more comprehensive U133 gene expression platform. Thus, U133A expression data from the NCI-60 panel of cell lines, chosen using identical approaches as previously described[4], was used to develop robust, refined gene expression signatures of chemosensitivity. Using an independent dataset $(n=133)$ of breast cancer tumor samples from patients treated neoadjuvantly with combination (TFAC) chemotherapy, we validated the performance of the individual predictors of sensitivity to paclitaxel (T), 5-fluorouracil (F), adriamycin (A), and cyclophosphamide $(\mathrm{C})$, as well as a combined predictor of TFAC sensitivity. We also use microRNA data from the NCI-60 cell lines to develop microRNA gene expression profiles of chemosensitivity, in an attempt to understand the biologic interplay between relevant microRNA expression in conjunction with the corresponding messenger RNA data, which could potentially refine the predictive ability of gene signatures. Finally, we use signatures of deregulated oncogenic signaling pathways in breast tumors to develop a strategy that identifies opportunities for other novel therapeutic drugs in patients resistant to chemotherapy, using the cohort of patients treated with TFAC as an example.

\section{Results}

mRNA signatures of paclitaxel, 5-fluorouracil, adriamycin, and cyclophosphamide sensitivity

Using identical cell lines identified previously as representing the extremes of sensitivity, gene signatures representative of sensitivity to paclitaxel, 5-fluorouracil, adriamycin, and cyclophosphamide were developed. The leave-one-out cross-validation probabilities for each chemotherapy sensitivity signature showed separation of sensitive and resistant cell lines with an accuracy always exceeding 95\% (Figure S1). The heatmaps (Fig la) of the probability values of the top genes used for prediction showed a clear demarcation between sensitive and resistant cell lines and the genes that were upregulated or downregulated in each.

The gene ontology of the genes used in each predictor, as discovered by performing a Batch Query on the Affymetrix website (www.affymetrix.com), included a plethora of genes and pathways thought to be important in cancer pharmacology and biology (Table S1). Genes used in predictors included those key in cell cycle regulation, signal recognition, tumor necrosis factor pathways, and growth arrest. Of note, the paclitaxel sensitivity signature included the Jun proto-oncogene, while the adriamycin signature included multiple members of the Ras-associated domain family as well as the epidermal growth factor receptor (EGFR) gene. The 5-fluorouracil signature included genes for transcription regulation and a cyclin-dependent kinase inhibitor (CDKN2A) thought to play a role in cancer progression, while the cyclophosphamide signature included genes (e.g. SET) commonly involved in leukemogenesis. Together, the ontology of the genes that constitute the four signatures reaffirms our confidence in their predictive ability.

\section{MicroRNA profiles characterize biology underlying chemotherapeutic resistance}

As a further step to advance our understanding of gene expression phenotypes of chemotherapeutic sensitivity and to begin to understand the role of microRNAs in predicting chemosensitivity, we also made use of relevant microRNA data from the individual NCI-60 cell lines. The heatmaps representing the microRNA chemosensitivity profiles showed demarcations between chemosensitive and resistant cell lines similar to the mRNA predictors (Figure 1b). Of particular interest is the fact that many of the individual microRNAs implicated in the individual profiles of chemosensitivity have previously been shown to have altered expression in human tumors (Table S2). Interestingly, miR-34, which was recently reported to mimic TP53 activity $[8,9,10,11,12]$, was downregulated in the sensitive cell lines in the 5-fluorouracil and paclitaxel microRNA signatures, indicating a possible connection between chemosensitivity to those drugs and the well-known TP53 tumor suppressor network. The fact that miR-34 was downregulated in cell lines sensitive to paclitaxel was surprising, as mutations in the TP53 pathway are thought to

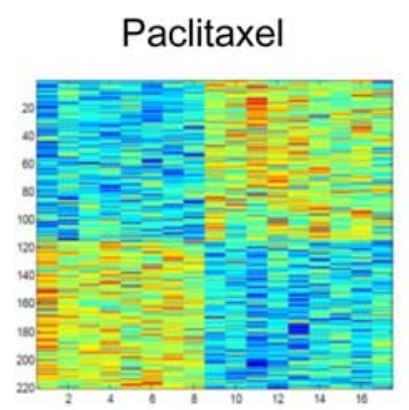

b

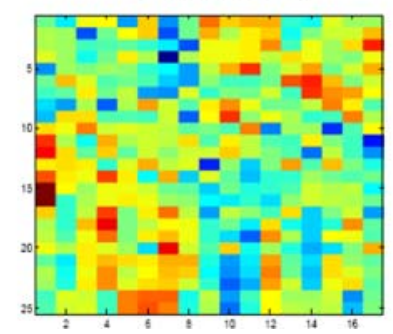

Fluorouracil

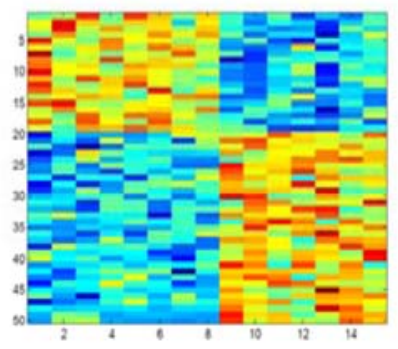

Fluorouracil

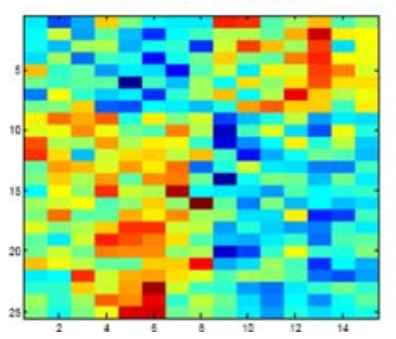

Adriamycin

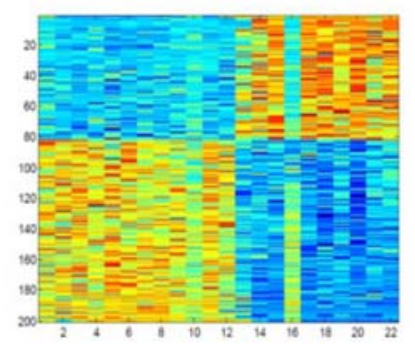

Adriamycin

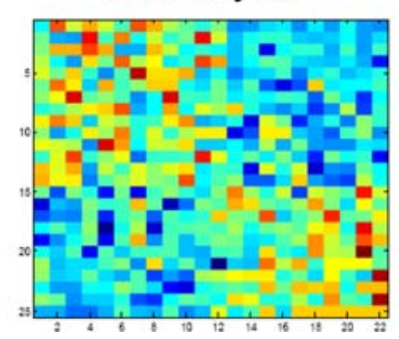

\section{Cyclophosphamide}

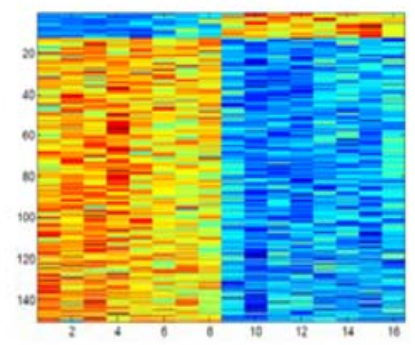

Cyclophosphamide

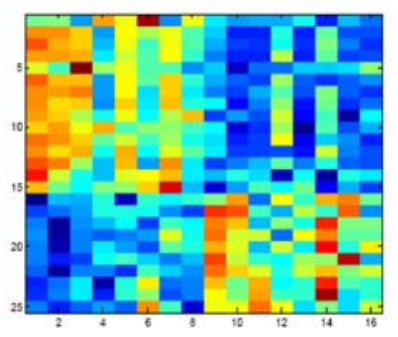

Figure 1. mRNA (a) and miRNA (b) gene signatures of sensitivity to paclitaxel, 5-fluorouracil, adriamycin, and cyclophosphamide. Gene number is displayed on the vertical axes, while sample number is listed horizontally. doi:10.1371/journal.pone.0001908.g001 


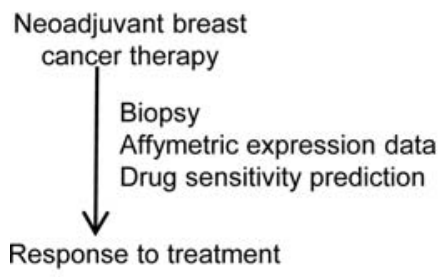

b

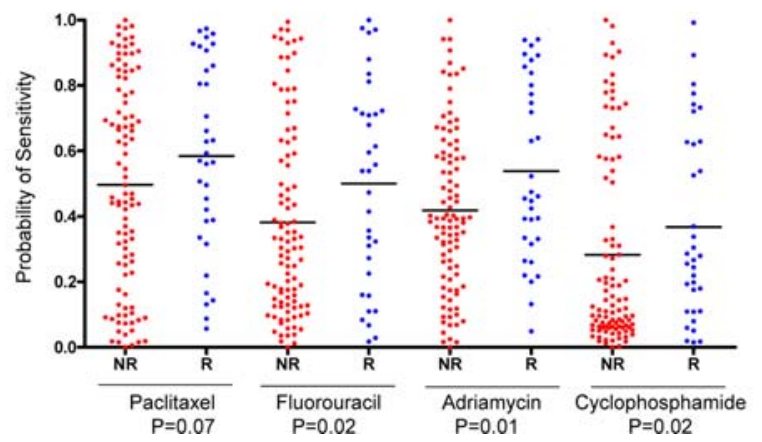

C

TFAC Combined Probability Values

Figure 2. Development and validation of chemotherapeutic response predictors. (a) Strategy for generating the chemotherapeutic response predictors. (b) Prediction of single-agent chemotherapy response in patient breast samples. Probability values of non-responders (NR) are shown in red, while probability values of responders (R) are shown in blue. Response was defined as complete pathologic response upon completion of TFAC neoadjuvant therapy. (c) Combined prediction of sensitivity to the TFAC chemotherapy regimen separated by non-responders ( $\mathrm{n}=99$, red) and responders $(n=34$, blue).

doi:10.1371/journal.pone.0001908.g002

confer resistance to taxanes[13]. This finding suggests that, while the inhibitory pathways of TP53 include abnormal tubulin detection and subsequent cell cycle arrest, miR-34 follows a separate path toward tumor suppression. In addition, miR-17 was downregulated in cell lines sensitive to adriamycin and cyclophosphamide, drugs commonly used in the treatment of lymphoproliferative disorders. The downregulation of miR-17 may represent an explanation for the effectiveness of these drugs in specific hematologic malignancies that are dependent on Myc pathway deregulation for their pathogenesis (e.g. chronic lymphocytic leukemia and aggressive lymphomas), since miR-17 functions synergistically with Myc to promote aggressive tumor growth in lymphoma[14]. Another microRNA thought to be involved with the Myc pathway, let-7, was implicated in the cyclophosphamide sensitivity signature in our analysis. Further, miR-let-7a, which has been shown to repress expression of the Myc transcription factor[15], was found to be downregulated in the cell lines sensitive to cyclophosphamide, an alkylating agent. These findings would imply that, if let-7 is downregulated in sensitive cell lines, Myc is upregulated, but the resultant proliferation is tempered by the repressive action of cyclophosphamide on cell replication. Lastly, miR-200b, which when inhibited confers sensitivity to chemotherapy in malignant cholangiocytes[16], was also found in our signature to be downregulated in cells sensitive to adriamycin, the current standard of care in the treatment for hepatobiliary tumors. MiR-200b suppresses a tumor suppressor gene (PTPN12) which is thought to inactivate the common oncogenes Src and Ras[16], such that the downregulation of miR-200b in sensitive cell lines allows the resultant proliferating cells to be targeted by adriamycin, a Topoisomerase II inhibitor. These findings suggest that, when utilized in an integrated approach, microRNA expression profiles in conjunction with corresponding gene expression data may provide a critical link for understanding mechanisms involved in chemosensitivity and chemoresistance.

\section{Validation of mRNA predictors in independent patient samples}

The true clinical value of gene expression signatures lies in their ability to predict response in large patient populations. Using previously developed methods to identify cell lines from the NCI60 that represent the extremes of drug sensitivity and the corresponding updated Affymetrix U133A data, we developed genomic predictors of TFAC sensitivity [4,6]. This study thus represents a refined predictive ability due to the use of U133A as opposed to the U95 data previously used. An independent dataset of 133 breast cancer tumor samples was used to evaluate the predictive ability of the U133A T, F, A, and C classifiers. When the predictive probability values of non-responders and responders to each individual chemotherapy agent were plotted, the median probability of sensitivity of the responders was higher than that of the non-responders (Figure 2) in each case. The probability of 5fluorouracil $(p=0.02)$, adriamycin $(p=0.01)$, and cyclophosphamide $(p=0.02)$ sensitivity were all significantly different between responders and non-responders in a Mann-Whitney U test, and the paclitaxel probability values showed a predictive trend, although not statistically significant $(p=0.07)$. Importantly, when the combined TFAC probability was plotted, the probability of sensitivity in the responders $(n=34$, Figure 2$)$ was significantly higher than the probability of sensitivity of the non-responders ( $\mathrm{n}=99, \mathrm{p}=0.002$, Mann-Whitney). The sensitivity of the combined predictor was 59\%, while the specificity was $63 \%$. Importantly, the negative predictive value, which is arguably the most relevant in a clinical scenario, as it should implicate patients who will not benefit from the treatment in question and should therefore be considered for other therapeutic options, was found to be $82 \%$.

\section{Effect of molecular variables on predicted chemosensitivity patterns}

Because molecular variables such as ER, PR, and HER2 status, as well as Topoisomerase IIA expression, have been shown to have an effect on chemotherapy sensitivity[17,18], we tested the effect of these molecular variables on the ability of gene signatures to accurately identify patients sensitive to TFAC therapy. While the prediction was significantly more robust in ER and HER negative patients, PR status had no major effect on TFAC prediction (Figure 3). When we separated the patients based on 'basal-like' (ER, HER, and PR negative) or 'non basal-like' status, the predictive ability of the TFAC combined signature was significantly greater in the basal-like patients (Figure 3). It may be important to note that the basal-like patients also showed a much higher likelihood of response (48\%) as opposed to the non basallike patients $(19 \%)$, as might be expected based on previous findings reported in the literature [18]. 
Effect of basal-like status on TFAC prediction

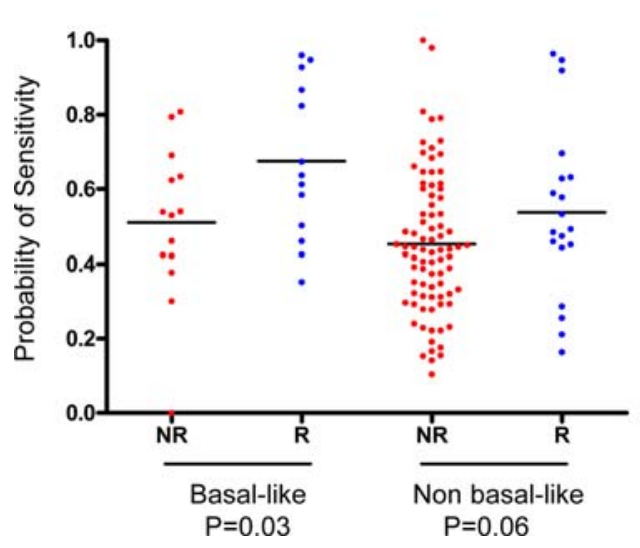

\section{.}

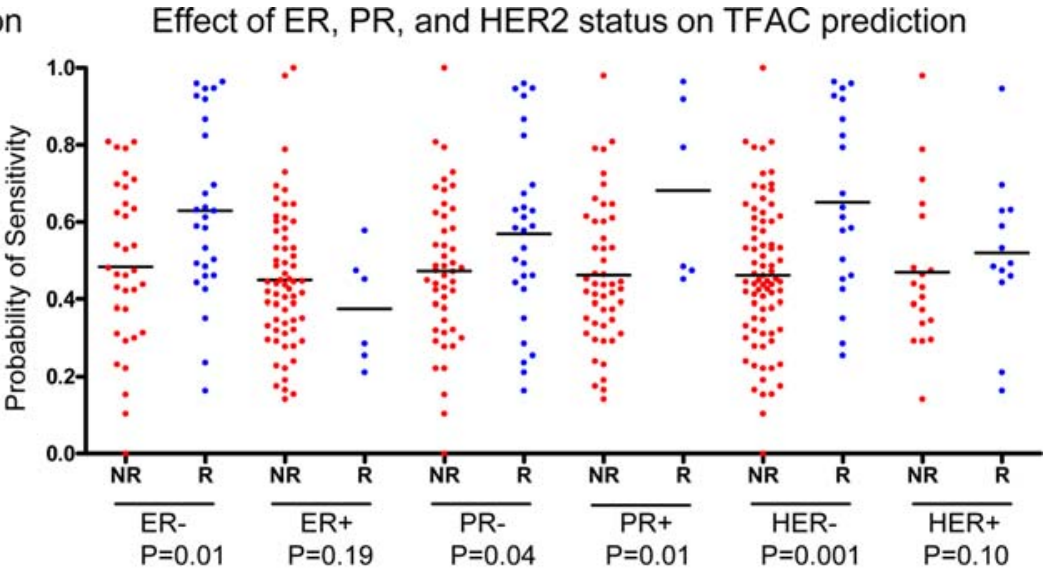

Effect of ER, PR, and HER2 status on TFAC prediction

Figure 3. Effect of molecular variables on combined TFAC prediction. Left, TFAC probability values of basal-like (HER2, ER, and PR negative) and non basal-like patients as separated by non-responders (NR) and responders (R). Right, TFAC probability values of non-responders and responders separated by ER score less than or greater than 50, PR status, and HER2 status.

doi:10.1371/journal.pone.0001908.g003

Likewise, because adriamycin is known to be a Topoisomerase IIA inhibitor, and HER2 status has been linked to Topoisomerase IIA expression[19], we analyzed the effects of HER2 status and Topoisomerase IIA expression independently on the predictive ability of the adriamycin sensitivity signature in the 133 patients. HER2 status seemed to have no major effect on adriamycin prediction, as both HER 2 positive and negative groups of patients showed a significant difference between the medians of the nonresponders and responders (Figure 4). Topoisomerase IIA expression, however, had a major effect on adriamycin prediction. When samples were divided into those with Topoisomerase IIA expression above and below the median value for each probe, the predictor for adriamycin sensitivity was significantly more robust in the samples with high Topoisomerase IIA expression as compared to low Topoisomerase IIA expression ( $p$ values of 0.0021 and 0.0022 versus 0.49 and 0.46 , respectively, Figure 4). Thus, it is likely that stratifying patients first by Topoisomerase IIA expression before the application of genomic predictors of chemotherapeutic response may improve the ability of the classifiers in predicting clinically significant response to adriamycin.

More broadly, this indicates a potential strategy to refine the predictive ability of gene expression-based signatures of chemosensitivity in breast cancer by first identifying cohorts based on relevant tumor biology such as ER, HER2 and Topoisomerase IIA expression.

Patterns of oncogenic pathway activation and alternative chemotherapeutic options in patients resistant to standard chemotherapy

The ability to accurately identify patients resistant to standard chemotherapy also emphasizes the need to dissect cancer biology further and identify alternative therapeutic strategies for patients resistant to therapy. To this end, we employed gene signatures representative of E2F, PI3K, Myc, $\beta$-catenin, Src, and Ras

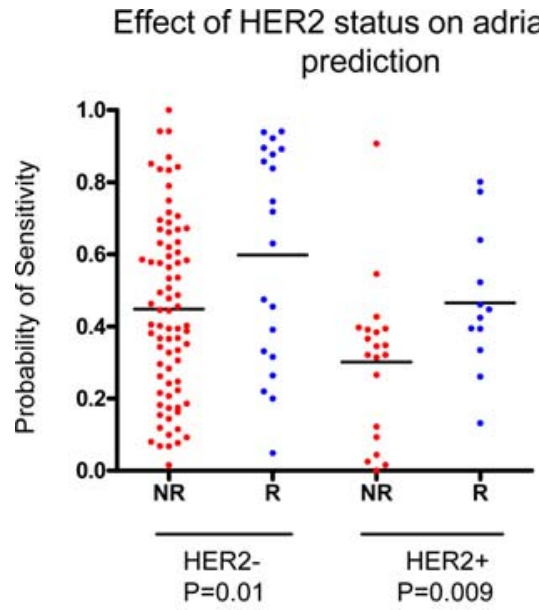

\section{Effect of Topoisomerase IIA expression on adriamycin prediction}

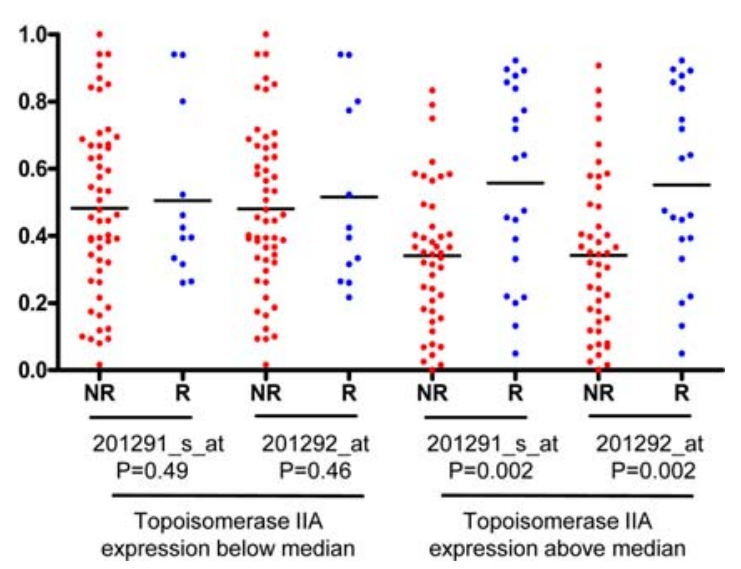

Figure 4. Effect of HER2 status and Topoisomerase IIA expression levels on adriamycin prediction. Left, patients were divided depending on HER2 negative or positive status, and predictive probability values of sensitivity to adriamycin were plotted for non-responders (NR) and responders (R). Right, patients were divided on the basis of whether their expression of Topoisomerase IIA (obtained using two different probes, 201291_s_at and 201292_at, in the U133A platform) was above or below the median value. Non-responders and responders were separated and their predictive probability values plotted.

doi:10.1371/journal.pone.0001908.g004 
pathway activation, as well as signatures of response to other commonly used cytotoxic agents in breast cancer (docetaxel, etoposide, vinorelbine and cisplatin), in the cohort of 133 patients.

Interestingly, none of the oncogenic signaling pathways alone were associated with the likelihood of response to TFAC therapy (Figure S2). While the knowledge gained from investigating individual pathways is sometimes critical in elucidating clinically relevant biology, breast cancer represents a very heterogeneous disease, the complexity of which may only be dissected by evaluating the oncogenic cooperation that exists between different signaling pathways. An approach to evaluating patterns of oncogenic cooperation between pathways is to use clustering strategies to demonstrate meaningful interactions between biologically relevant mechanisms. As shown in Figure 5, unsupervised hierarchical clustering of the oncogenic pathways E2F, PI3K, Myc, $\beta$-catenin, Src, and Ras in the cohort of 133 patients demonstrated significant, biologically relevant patterns of pathway activation. Importantly, analysis comparing the percent of responders in each cluster showed that two clusters had significantly higher or lower responder percentages (Figure 5). Whereas the entire population of patients was comprised of $25 \%$ responders, Cluster 3 had a much higher percentage of responders at $54 \%$, and Cluster 4 had a lower population of responders at only $8 \%$. Clusters 1, 2, and 5 had numbers of responders not significantly different from the entire population. A t-test between Clusters 3 and 4 showed that they were significantly different $(p=0.003$, Mann-Whitney). In the interest of examining the two extremes of sensitivity, Clusters 3 and 4 were compared for further analyses. While Cluster 3, with the higher percentage of responders, seemed to show predominant activation of the Myc and Ras pathways, Cluster 4 showed activation of the Myc and E2F pathways. Therefore, we can hypothesize that E2F activation, in combination with Myc as opposed to Ras activation, may confer resistance to TFAC chemotherapy in patients with breast cancer.

Further, to identify alternative chemotherapeutic options in patients resistant to TFAC therapy (Cluster 4), predictive probabilities of sensitivity to cisplatin, docetaxel, etoposide, and vinorelbine (Figure 5), all agents commonly used to treat breast cancer, were generated using U133A data. Interestingly, the most distinctive characteristic of Cluster 4 was the high probability of sensitivity to docetaxel (Figure 5). Importantly, a predictor of docetaxel sensitivity was also validated $(p=0.02$, Figure S3) in an independent dataset of 24 patients treated neoadjuvantly with the single agent docetaxel[20]. This provided us with the opportunity to compare a robust predictor of docetaxel sensitivity across the current dataset of 133 patients that were clustered based on their oncogenic pathway activation status. When compared to patients in other clusters, the non-responders in Cluster 4 had a significantly greater probability of responding to docetaxel $(\mathrm{P}<0.001$, Figure S4). Furthermore, when the probability of sensitivity to docetaxel was plotted against E2F activation in nonresponders to chemotherapy, a trend towards a positive correlation was observed $(\mathrm{P}=0.07$, Figure $\mathrm{S} 4)$. This implies that patients with an activated E2F pathway that are resistant to TFAC may benefit from the alternative treatment of docetaxel. Previous studies[21] showed that head and neck squamous cell carcinoma samples that were resistant to cisplatin and sensitive to docetaxel (similar to our Cluster 4 tumor samples) showed decreased E2F activation after treatment with docetaxel, which further supports the argument that the subset of patients in Cluster 4 may benefit from alternative treatment with docetaxel. The finding of the relationship between E2F and docetaxel is interesting biologically and emphasizes the importance of a validated predictor of docetaxel sensitivity in patients with early stage breast cancer. To this extent, we have employed independent data from Chang J et al [20], a study involving the neoadjuvant use of single agent docetaxel in patients with breast cancer. As shown in Figure S3, a U133A predictor of docetaxel (Table S3) accurately separates responders from non-responders $(\mathrm{P}=0.02$, sensitivity: $90 \%$, specificity: $57 \%$, positive predictive value: $60 \%$, negative predictive value: $89 \%$, Figure S3).

It is important to emphasize that the results shown here represent an example of the complexity involved in defining phenotype of chemosensitive or chemoresistant disease. Knowledge of clinico-pathologic variables likely to affect an individual patient's response to a particular drug or regimen will only lead to

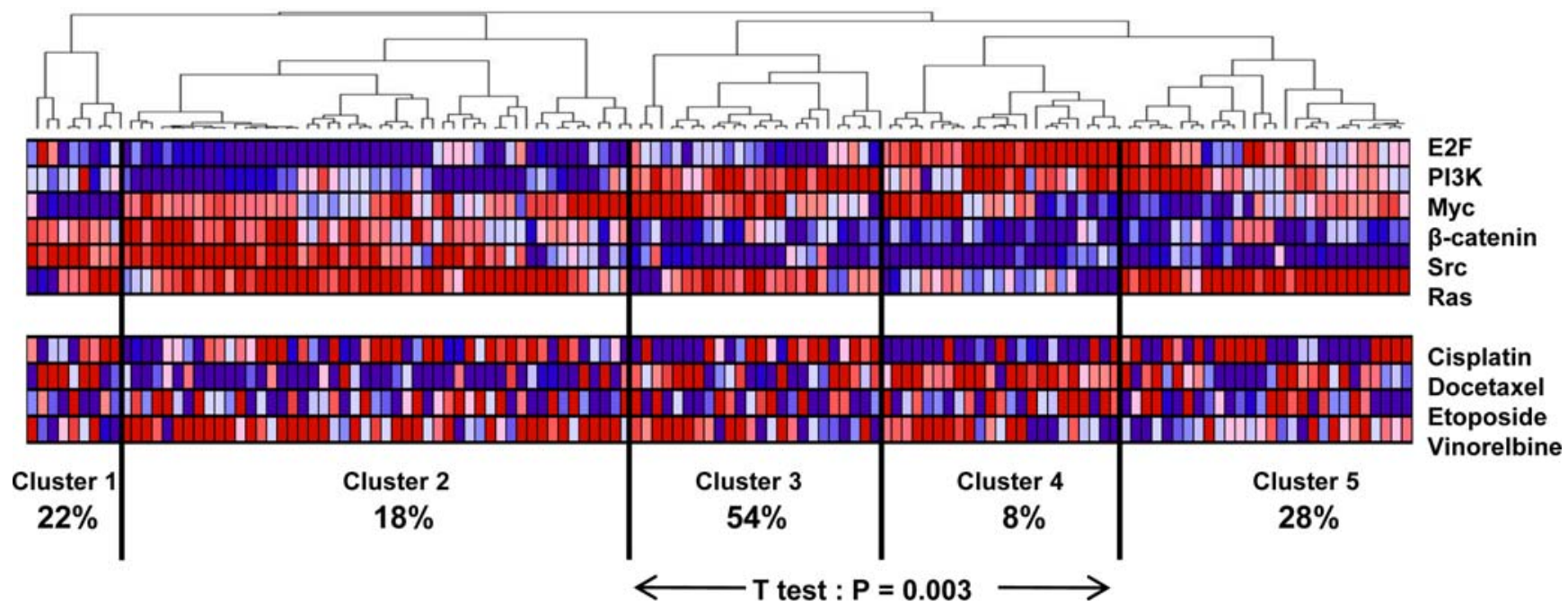

Figure 5. Patterns of predicted oncogenic pathway activation and alternative chemotherapeutic options in human breast cancer tumors. Above, hierarchical clustering of a collection of breast tumors $(n=133)$ according to patterns of oncogenic pathway activation. Below, predictions of sensitivity to other commonly used chemotherapeutic agents in breast tumors. Predictions were plotted as heatmaps in which a high probability of sensitivity (or response) is indicated by red, and low probability (or resistance) is indicated in blue. The percentage of responders in each cluster is reported.

doi:10.1371/journal.pone.0001908.g005 
the application of gene signatures in a more effective and refined matter.

\section{Discussion}

The selection of the right chemotherapeutic agent for individual cancer patients remains a challenge. Chemotherapy-associated morbidity is a major concern, and strategies to limit therapies used to those that are most likely to be effective have the potential to change the current paradigm of cancer treatment. The results we present here provide further evidence that the use of gene expression data to predict chemotherapy response and oncogenic pathway activation may assist in selecting therapeutics on a patient-by-patient basis. While the cell lines used and methods described are identical to previously validated signatures of sensitivity, we believe that the development of robust U133Aplatform gene expression signatures of chemosensitivity may be a very useful tool in further validation strategies and eventually in guiding patient treatment. Importantly, the paclitaxel, 5-fluorouracil, adriamycin, and cyclophosphamide signatures developed showed clinically relevant predictive ability in a population of human breast tumor samples obtained from patients treated neoadjuvantly with TFAC therapy.

Beyond the development of U133A mRNA signatures for chemosensitivity, we also present data to suggest that microRNA expression may be useful in further dissecting the phenomenon of chemotherapy resistance and in predicting patterns of sensitivity. This finding is not very surprising, given the fact that microRNAs can function as either tumor suppressors or as oncogenes, and that altered microRNA levels are found in various human cancers [22,23]. Notably, although preliminary, these profiles are the first to use microRNA data to constitute signatures of chemosensitivity and resistance. Thus, pending further validation, our results present preliminary data supporting the hypothesis that microRNA signatures may be used to complement mRNA gene expression signatures of chemosensitivity.

While the chemotherapy sensitivity signatures described are useful for predicting which patients will respond to a specific regimen (TFAC), used alone they do not address the important issue of alternative therapeutic options for patients who would not respond to TFAC. Here, we use TFAC as a representative example of the bigger challenge in oncology with respect to chemotherapy, the issue of viable options for non-responders to traditional chemotherapeutic regimens. For this reason, we used signatures of oncogenic pathway activation[24] as well as signatures for other commonly used chemotherapy agents in breast cancer (cisplatin, docetaxel, etoposide, and vinca alkaloids) to identify potentially activated pathways that might be future targets for therapy, while also suggesting alternative chemotherapeutic options. Our data suggest that, in the case of Cluster 4 (which had the lowest percentage of responders) the activated E2F pathway may be driving chemoresistance, and alternative treatment with a regimen including docetaxel may have resulted in an increased number of responders. Importantly, the oncogenic pathway activation signatures employed were unable alone to predict responder status of patients, but combined with alternative chemotherapeutic signatures, they suggest a cohort of patients to that may benefit from alternative treatment. While our body of data represents a total of 157 patients, additional studies will be needed to confirm the trends observed, especially the benefit of docetaxel treatment in E2F-activated breast cancer cell lines resistant to other commonly used agents.

In conclusion, the reported chemotherapy sensitivity signatures can be used effectively in the prediction of response, while other predictors of oncogenic pathway activation [24] and even tumor microenvironment should add information that further improves our understanding of cancer biology while also leading to alternative therapeutics for predicted non-responders. Importantly, relevant molecular or pathologic information may aid our understanding of chemotherapy resistance and sensitivity in breast cancer. It is likely that the most optimal approach to prediction of response to cytotoxic therapy will involve a combination of gene expression data, microRNA profiles, and molecular variables such as ER, HER2, and PR status. The goal is to develop a strategy to determine a personalized treatment approach to breast cancer, so that each individual patient will have a better chance of a favorable outcome by matching specific treatment options to their molecular profiles.

\section{Methods}

\section{Development of mRNA and microRNA signatures}

The NCI-60 cell lines that were most resistant or sensitive to each chemotherapy agent were identified as previously described[4], and the Affymetrix HG-U133A based NCI-60 cell line expression data was obtained from the National Institute of Health (courtesy John Weinstein). Mas5 expression values were $\log 2$ transformed, and class labels (zero or one) were assigned to sensitive and resistant cell lines. The predictors were optimized for performance by applying multiple t-tests with a cut-off probability value of 0.01 using statistical package $R$ [25] (version 2.5.1). Using methods previously described[4,24], top gene probe identifiers that best separate sensitive from resistant cell lines were identified. Leave-one-out cross-validation, in which each cell line is removed from the signature one-by-one and its predictive probability value generated using the remaining cell lines, was used to assess the accurate and robust nature of each individual predictor. As a proof-of-concept approach to combining probabilities, the TFAC predictor was generated by taking the mean of the mean-centered probability values generated by the four individual chemotherapy sensitivity signatures.

The same NCI-60 cell lines selected for the Affymetrix HGU133A-based mRNA signatures were used to develop microRNA signatures of chemotherapy sensitivity and resistance. MicroRNA pin-spotted expression data was downloaded from the Cell Miner database in .gpr format. Using GeneSpring (Agilent), the signal intensity of each spot was calculated by subtracting local background (based on the median intensity of the area surrounding each spot) from the median signal. As a means of nonspecific filtering, raw values used were limited to those between 8 and 65. Signal intensities were $\log 2$ transformed and duplicate spots were averaged. Quantile normalization was performed across the 3 microarray batches, all values $<5$ were replaced with the median of such values, and the value for each control cell line was set to the mean of its five replicates. All control and mouse probes were removed, and only the resulting 273 human microRNA probes were used in remaining analyses. Bayesian binary regression performed in MatLab identified the top gene probe identifiers used in separating resistant from sensitive cell lines, and those probes are reported in Table S2.

\section{Patients and Samples}

A validation set of 133 human breast cancer samples was obtained from the MD Anderson Cancer Center website (http:// bioinformatics.mdanderson.org/pubdata.html). This dataset was selected due to its clinically annotated data (Affymetrix Human Genome U133A) for patients treated with neoadjuvant chemotherapy, an ideal setting to evaluate the efficacy of gene 
expression-based predictors of response. All patients were treated with 24 weeks of sequential paclitaxel and fluorouracil-adriamycin-cyclophosphamide neoadjuvant chemotherapy, except two patients who were treated with either only paclitaxel or a combination of paclitaxel, 5-fluorouracil, epirubicin, and cyclophosphamide[26]. The clinical data designated patients as either non-responders $(n=99)$ or responders $(n=34)$ on the basis of pathologic complete response; in other words, responders showed no signs of residual invasive cancer in the breast or lymph nodes at the time of surgery. HER2 status, ER status (based on ER score), and PR status were obtained from the clinical data provided on the M.D. Anderson website and used in the analysis of the effect of molecular variables on chemotherapy sensitivity prediction. 'Basallike' patients were defined as patients with negative ER, HER2, and PR status. Topoisomerase IIA expression was obtained from the $\log 2$-transformed expression data from two representative probes, 201281_s_at and 201292_at, and divided into values above and below the median. For the docetaxel validation, an independent dataset of 24 samples treated neoadjuvantly with docetaxel was used[20].

\section{Application of oncogenic pathway activation and alternative chemotherapeutic agent signatures}

Oncogenic pathway signatures described previously[24] were used to create predictive probability values for activation of the $\beta$ catenin, E2F, Myc, PI3K, Ras, and Src pathways. The pathway signatures, combined with the validation set of breast tumor samples, were standardized using MatLab (version 7.1). Bayesian probit binary regression analysis was then performed on the datasets, and the resultant probability values generated. Unsupervised hierarchical clustering was performed using complete linkage clustering with Pearson correlation coefficient in the Gene Pattern[27](Hierarchical Clustering module, version 3.0) software package, and a heatmap was then generated. Clusters were assigned by visualization of the corresponding dendrogram, and the percent of responders in each cluster were calculated.

Genomic signatures for docetaxel, etoposide, cisplatin, and a vinca alkaloid (a surrogate for vinorelbine) were applied to the breast tumor validation set to identify alternative chemotherapeutic options for non-responders to TFAC. The signatures for docetaxel and etoposide were developed as described above for the individual TFAC predictors. Briefly, cell lines selected from the NCI-60 for the Affymetrix HG-U95 based Av2 predictors[4] were used in the HG-U133A-based docetaxel and etoposide predictors, multiple t-tests were applied on the dataset using statistical package $\mathrm{R}$ (version 2.5.1), and the binary regression parameters were adjusted to achieve optimal cross-validation accuracy. The signatures for cisplatin and vinca alkaloid were developed using a set of 30 human cancer cell lines[28]. Resultant predictive probability values were displayed as a heatmap using HeatmapViewer module of the GenePattern software based on patient sample order obtained earlier from the unsupervised hierarchical clustering of the oncogenic pathway activation probability values. Clusters previously defined based on the patterns of oncogenic pathway activation were analyzed to assess whether alternative chemotherapeutic options were likely to be effective for specific

\section{References}

1. Staunton JE, Slonim DK, Coller HA, Tamayo P, Angelo MJ, et al. (2001) Chemosensitivity prediction by transcriptional profiling. Proc Natl Acad Sci U S A 98: 10787-19792.

2. Herbst RS, Bajorin DF, Bleiberg H, Blum D, Hao D, et al. (2006) Clinical Cancer Advances 2005: major research advances in cancer treatment, prevention, and screening-a report from the American Society of Clinical Oncology. J Clin Oncol 24: 190-205. patient clusters predicted to be predominantly resistant to the original chemotherapy agent.

\section{Supporting Information}

Figure S1 mRNA gene signatures of sensitivity to paclitaxel, 5fluorouracil, adriamycin, and cyclophosphamide. Heatmaps, with gene number on the horizontal axis and sample number on the vertical axis, are shown above. Below, leave-one-out crossvalidation of samples selected to represent resistance (blue) and sensitivity (red) to each chemotherapeutic agent.

Found at: doi:10.1371/journal.pone.0001908.s001 (4.86 MB TIF)

Figure S2 Individual oncogenic pathway signatures as applied to the cohort $(n=133)$ of breast cancer patients. Non-responders and responders were separated and their predictive probability values plotted.

Found at: doi:10.1371/journal.pone.0001908.s002 (2.05 MB TIF)

Figure S3 Validation of a U133A predictor of docetaxel sensitivity in an independent dataset of 24 samples from patients treated neoadjuvantly with single agent docetaxel

Found at: doi:10.1371/journal.pone.0001908.s003 (1.21 MB TIF)

Figure S4 Docetaxel sensitivity and E2F activation in TFAC non-responders. The predicted probability of sensitivity to docetaxel of the TFAC non-responders as divided by individual clusters (Panel A) and Cluster 4 compared with the other clusters (Panel B). Panel $\mathrm{C}$ shows a linear regression analysis of the probability of sensitivity to docetaxel plotted against the E2F oncogenic pathway activation.

Found at: doi:10.1371/journal.pone.0001908.s004 (2.37 MB TIF)

Table S1 Ontology of genes used in the TFAC chemotherapy sensitivity signatures.

Found at: doi:10.1371/journal.pone.0001908.s005 (0.57 MB DOC)

Table S2 Ontology of miRNA genes used in chemotherapy sensitivity signatures.

Found at: doi:10.1371/journal.pone.0001908.s006 (0.07 MB DOG)

Table S3 Ontology of genes used in the docetaxel sensitivity signature.

Found at: doi:10.1371/journal.pone.0001908.s007 (0.21 MB DOC)

\section{Acknowledgments}

The authors thank the research support from the Jimmy V foundation and the Emilene Brown Cancer Research Fund.

\section{Author Contributions}

Conceived and designed the experiments: JN JO AP KM KS. Performed the experiments: CA KS. Analyzed the data: SM HD AP CA KS KW WB. Contributed reagents/materials/analysis tools: SM HD JN JO AP CA WB. Wrote the paper: JN AP KG KM KS KW RR AA. Other: Designed the study: AP.

3. Breathnach OS, Freidlin B, Conley B, Green MR, Johnson DH, et al. (2001) Twenty-two years of phase III trials for patients with advanced non-small-cell lung cancer: sobering results. J Clin Oncol 19: 1734-1742.

4. Potti A, Dressman HK, Bild A, Riedel RF, Chan G, et al. (2006) Genomic signatures to guide the use of chemotherapeutics. Nat Med 12: 1294-1300.

5. Nevins JR, Potti A (2007) Mining gene expression profiles: expression signatures as cancer phenotypes. Nat Rev Genet 8: 601-609. 
6. Bonnefoi H, Potti A, Delorenzi M, Mauriac L, Campone M, et al. (2007) Validation of gene signatures that predict the response of breast cancer to neoadjuvant chemotherapy: a substudy of the EORTC 10994/BIG 00-01 clinical trial. Lancet Oncol.

7. Hsu DS, Balakumaran BS, Acharya CR, Vlahovic V, Walters KS, et al. (2007) Pharmacogenomic strategies provide a rational approach to the treatment of cisplatin-resistant patients with advanced cancer. J Clin Oncol 25: 4350-4357.

8. Chang TC, Wentzel EA, Kent OA, Ramachandran K, Mullendore M, et al. (2007) Transactivation of miR-34a by p53 broadly influences gene expression and promotes apoptosis. Mol Cell 26: 745-752.

9. Bommer GT, Gerin I, Feng Y, Kaczorowski AJ, Kuick R, et al. (2007) p53mediated activation of miRNA34 candidate tumor-suppressor genes. Curr Biol 17: 1298-1307.

10. He L, He X, Lim LP, de Stanchina E, Xuan Z, et al. (2007) A microRNA component of the p53 tumour suppressor network. Nature 447: 1130-1134.

11. Raver-Shapira N, Marciano E, Meiri E, Spector Y, Rosenfeld N, et al. (2007) Transcriptional activation of miR-34a contributes to p53-mediated apoptosis. Mol Cell 26: 731-743.

12. Tarasov V, Jung P, Verdoodt B, Lodygin D, Epanchintsev A, et al. (2007) Differential regulation of microRNAs by p53 revealed by massively parallel sequencing: miR-34a is a p53 target that induces apoptosis and G1-arrest. Cell Cycle 6: 1586-1593.

13. Dumontet C, Sikic BI (1999) Mechanisms of action of and resistance to antitubulin agents: microtubule dynamics, drug transport, and cell death. J Clin Oncol 17: 1061-1070.

14. Tagawa H, Karube K, Tsuzuki S, Ohshima K, Seto M (2007) Synergistic action of the microRNA-17 polycistron and Myc in aggressive cancer development. Cancer Sci 98: 1482-1490.

15. Park SM, Shell S, Radjabi AR, Schickel R, Feig C, et al. (2007) Let-7 prevents early cancer progression by suppressing expression of the embryonic gene HMGA2. Cell Cycle 6: 2585-2590.

16. Meng F, Henson R, Lang M, Wehbe H, Maheshwari S, et al. (2006) Involvement of human micro-RNA in growth and response to chemotherapy in human cholangiocarcinoma cell lines. Gastroenterology 130: 2113-2129.
17. Hayes DF, Thor AD, Dressler LG, Weaver D, Edgerton S, et al. (2007) HER2 and response to paclitaxel in node-positive breast cancer. N Engl J Med 357: 1496-1506.

18. Rouzier R, Perou CM, Symmans WF, Ibrahim N, Cristofanilli M, et al. (2005) Breast cancer molecular subtypes respond differently to preoperative chemotherapy. Clin Cancer Res 11: 5678-5685.

19. Mano MS, Rosa DD, De Azambuja E, Ismael GF, Durbecq V (2007) The 17q12-q21 amplicon: Her2 and topoisomerase-IIalpha and their importance to the biology of solid tumours. Cancer Treat Rev 33: 64-77.

20. Chang JC, Wooten EC, Tsimelzon A, Hilsenbeck SG, Gutierrez MC, et al. (2003) Gene expression profiling for the prediction of therapeutic response to docetaxel in patients with breast cancer. Lancet 362: 362-369.

21. Yoo GH, Piechocki MP, Ensley JF, Nguyen T, Oliver J, et al. (2002) Docetaxel induced gene expression patterns in head and neck squamous cell carcinoma using cDNA microarray and PowerBlot. Clin Cancer Res 8: 3910-3921.

22. Calin GA, Dumitru CD, Shimizu M, Bichi R, Zupo S, et al. (2002) Frequent deletions and down-regulation of micro- RNA genes miR15 and miR16 at $13 q 14$ in chronic lymphocytic leukemia. Proc Natl Acad Sci U S A 99: $15524-15529$.

23. Volinia S, Calin GA, Liu GG, Ambs S, Cimmino A, et al. (2006) A microRNA expression signature of human solid tumors defines cancer gene targets. Proc Natl Acad Sci U S A 103: 2257-2261.

24. Bild AH, Yao G, ChangJT, Wang Q Potti A, et al. (2006) Oncogenic pathway signatures in human cancers as a guide to targeted therapies. Nature 439: 353-357.

25. Ihaka RaG, RE (1996) R: A Language for Data Analysis and Graphics. Journal of Computational and Graphical Statistics 5: 299-314.

26. Pusztai L, Anderson K, Hess KR (2007) Pharmacogenomic Predictor Discovery in Phase II Clinical Trials for Breast Cancer. Clin Cancer Res 13: 6080-6086.

27. Reich M, Liefeld T, Gould J, Lerner J, Tamayo P, et al. (2006) GenePattern 2.0. Nat Genet 38: 500-501.

28. Gyorffy B, Surowiak P, Kiesslich O, Denkert C, Schafer R, et al, (2006) Gene expression profiling of 30 cancer cell lines predicts resistance towards 11 anticancer drugs at clinically achieved concentrations. Int J Cancer 118: $1699-1712$. 